\title{
Amount of priming and the cue depreciation effect
}

\author{
ZEHRA F. PEYNIRCIOĞLU \\ The American University, Washington, D.C.
}

In word-fragment cuing tests, disclosing a test item piecemeal (e.g., r......p; r-...r-p; r-i--r-p; $r$-i--rop) rather than all at once (r-i--rop) inhibits its discovery. Such cue depreciation has thus far been observed only with items that were either studied beforehand or otherwise primed. In the present study, the effect of amount of priming on the magnitude of the inhibition was investigated. In Experiment 1, some words were presented once and others were presented five times; in Experiment 2, some words were designated the to-be-remembered items and were studied with a deep orienting task, while others were designated the to-be-forgotten items and were studied with a shallow orienting task. In both experiments, the cue depreciation effect dissipated with less primed words, which suggests that priming per se is not the critical factor influencing inhibition.

In word-fragment cuing tests, if the cue for a word is disclosed gradually (e.g., r------p; r----r-p; r-i--r-p; $r-i-$-rop), the discovery of the word ("raindrop") is less likely than if the cue is disclosed all at once (r-i--rop). This is the case even though, with equal times allowed at each stage of cuing, subjects have actually more time to think in the former (incremental cue) condition than in the latter (standard cue) condition (Peynircioğlu \& Watkins, 1986). To date, such a cue depreciation effect has been observed only with just-presented items or with items that had been primed in some way to limit the target set; no effect has been observed with an unlimited set of general-knowledge items (e.g., Peynircioğlu, 1987).

The present study further explored the effect with primed items, focusing on just-presented items. More specifically, the question of interest was whether the magnitude of the inhibitory effect would vary with the amount of priming, and thus whether greater priming would induce a greater effect. To this end, in Experiment 1, in one long list of words, some words were presented once and others five times, and in Experiment 2, some words were designated the to-be-forgotten words and were studied with a shallow orienting task, and others were designated the to-be-remembered words and were studied with a deep orienting task. In both cases, the question of interest was whether the words that received greater priming (by virtue of being presented more times or studied with a deep orienting task) would show a greater cue depreciation effect.

\section{EXPERIMENT 1}

\section{Method}

Materials and Design. A total of 48 common eight-letter words were selected; a random 24 were assigned to the once-presented condition

Correspondence concerning this article should be addressed to Zehra F. Peynircioglu, Department of Psychology, The American University, 4400 Massachusetts Ave. N.W., Washington, DC 20016. and the other 24 were assigned to the five-times presented condition The words in the two conditions were interchanged between two groups of subjects. The presentation list included all 48 words. Thus, there were 144 items printed one under the other on a sheet of paper to be studied at a rate of one every $2 \mathrm{sec}$; presentation of repeated words were separated from each other by at least five intervening words.

A word-fragment cuing test followed the presentation list. For testing purposes, a two-letter fragment was constructed for each word which specified that word uniquely within the set of words that were used three-, four-, and five-letter fragments were constructed simply by adding letters to the initial two-letter fragment. These successively larger fragments for each word were printed one under the other on a single sheet of paper. A random half of the words in each repetition condition were tested in the incremental cue condition: for each word, first a twoletter fragment was shown, then other letters were added gradually to reveal the three-, four-, and five-letter fragments. The other half of the words were tested in the standard cue condition, in which the five-letter fragment for each word was shown right away, without going through the smaller fragments. The testing order of the words was different from the presentation order and was mixed with respect to cue condition. The answer sheets the subjects were given contained a box with four slots for each test word; each slot was reserved for each stage of cuing. In the incremental cue condition, $5 \mathrm{sec}$ were allowed at each stage of cuing, for a total of $20 \mathrm{sec}$ for each word, and in the standard cue condition, $5 \mathrm{sec}$ were allowed at the only stage of cuing for each word. Words tested in the incremental and standard cue conditions were interchanged between two subgroups of subjects within the two repetition-condition groups.

Procedure. The subjects were presented with a long list of eight-letter words to study, and were then given a memory test. Some words in the study list appeared more than once and others appeared just once. The subjects studied each word by reading down the columns of words printed on the sheet in front of them, paced by the experimenter. The memory test entailed discovering the studied words from their fragments. For some words, initially a very small fragment including only two letters was shown, then more letters were added until the fragment included five letters. This was accomplished by holding a cardboard cover in front of a test sheet and lowering it gradually to reveal the successively larger fragments of the target word. For other words, the cover was removed all at once and the final fragment including the five letters was shown right away. The answer sheets comprised a box with four slots for each word. In the incremental cue condition, if a word was discovered at the two-letter stage of cuing, it was to be written in the first slot, if it was discovered at the next stage, it was to be written in the second slot, and so on. A mistake in any slot was treated the same way as a slot left blank; thus, guessing was not penalized. In the standard cue condition, the subjects were asked to cross off the first three 
slots before the single large fragment was shown; if they discovered the word, they wrote it in the last slot.

Subjects. A total of 60 university students participated in the experiment for extra credit in psychology courses. They were tested in small groups.

\section{Results}

As expected, overall, a higher percentage of the fivetimes-presented words were discovered than once-presented words [66.6 vs. $51.3 ; t(59)=8.84, p<.01]$. Also, there was an overall cue depreciation effect: a lower percentage of fragments were completed in the incremental condition than in the standard cue condition [56.3 vs. 61.5 ; $t(59)=2.97, p<.01]$.

Of main interest, however, was whether the amount of priming affected cue depreciation. Thus, the oncepresented and five-times presented words were analyzed separately. Unexpectedly, inhibition was found only for the five-times presented words. On average, $62.6 \%$ of the fragments were completed in the incremental cue condition, compared to $70.6 \%$ in the standard cue condition $[t(59)=2.85, p<.01]$. Corresponding percentages for once-presented words were 50.0 and 52.5 , and this difference, although in the usual direction for primed items, was not reliable $[t(59)=0.92, p>.10]$. Although the interaction between repetition condition and cue condition was not reliable $[t(59)=1.28, p>.10]$, the cue depreciation effect with once-presented words was nevertheless suppressed.

\section{EXPERIMENT 2}

\section{Method}

The method was similar to that of Experiment 1. A total of 80 eightletter common words were presented in one long list at a rate of one word every $5 \mathrm{sec}$. Half of the words were written in red ink and the other half in black ink; the subjects were told that the memory test that would follow would include only the red words, and that the black words were there simply to distract them and lower their learning efficiency for the red words. Thus, the subjects were told to try to forget the black words and to rehearse and remember the red ones; to make remembering the red ones easier, the subjects were told to think about the meaning of each red word and to rate its pleasantness on a scale of 1-5, where 1 was "least pleasant" and 5 was "most pleasant." They were also told that they could not ignore the black words completely, because if they did there would be no distraction. Therefore, the subjects were instructed to count the number of consonants in each black word and to indicate whether it ended in a vowel or a consonant. All subjects were presented with all words in the same order, but for half of the subjects odd-numbered words were printed in red ink and were the to-beremembered words and even-numbered words were printed in black ink and were the to-be-forgotten words, and for the other half, vice versa. The test procedure was identical to that of Experiment 1.
A total of 80 university students participated in the experiment for extra credit in psychology courses. They were tested in small groups.

\section{Results}

Not surprisingly, there was an overall effect of orienting task and instruction (Craik \& Tulving, 1975); on average, $60.7 \%$ of the fragments of to-be-remembered words were completed, compared to $47.1 \%$ of those of to-beforgotten words $[t(79)=12.14, p<.01]$. Also, as before, an overall cue depreciation effect was present; on average, $51.5 \%$ of the fragments were completed in the incremental cue condition, compared to $56.2 \%$ in the standard cue condition $[t(79)=4.45, p<.01]$.

When the words in the two study conditions were analyzed separately, a result similar to that of Experiment 1 emerged. The cue depreciation effect was present only with the to-be-remembered words; on average, $56.9 \%$ of the fragments were completed in the incremental cue condition, compared to $64.4 \%$ in the standard cue condition $[t(79)=4.49, p<.01]$. The corresponding percentages with the to-be-forgotten words were 46.1 and 48.1 , and this difference, although in the expected direction for primed items, was not reliable $[t(79)=1.18, p>.10]$. Moreover, the cue depreciation effect with the to-beremembered words was reliably greater than that with the to-be-forgotten words $[t(79)=2.12, p<.025]$.

\section{CONCLUSION}

In this study, the amount of priming affected cue depreciation. What was somewhat surprising, though, was that rather than showing an increase in magnitude with the more primed items, the inhibitory effect dissipated with the relatively less primed items. Although, of course, caution is needed for comparisons across experiments, the size of the effect with the more primed items in both experiments was similar to that obtained when the rough equivalents of "less" primed items were tested among nonprimed items in comparable situations (cf. Peynircioğlu \& Watkins, 1986). Thus, it appears that the cue depreciation effect does not depend entirely on priming per se; it is also influenced by subjects' perceptions or interpretations.

\section{REFERENCES}

Craik, F. I. M., \& Tulving, E. (1975). Depth of processing and retention of words in episodic memory. Journal of Experimental Psychology: General, 104, 268-294.

PeYnircióclu, Z. F. (1987). Inhibition through fragment cuing with primed items. Journal of Experimental Psychology: Learning, Memory, \& Cognition, 13, 569-572.

Peynircioğlu, Z. F., \& Watkins, M. J. (1986). Cue depreciation: When word fragment completion is undermined by prior exposure to lesser fragments. Journal of Experimental Psychology: Learning, Memory, \& Cognition, 12, 426-431. 